\title{
LOS ESTUDIOS SOBRE EL NIVEL DE VIDA. LA METODOLOGÍA DE LA CANASTA APLICADA A LA PRIMERA MITAD DEL SIGLO XIX PORTEÑO
}

\author{
Studies on living standards. The methodology of consumption basket applied to \\ Buenos Aires, first half of 19th centuries
}

\section{Daniel Santilli* y Jorge Gelman**}

\section{Resumen}

En este trabajo se analiza la metodología de la construcción de canastas de consumo de las familias de Buenos Aires en la primera etapa independiente, a partir de modelos considerados válidos para la comparación internacional. Dichos modelos y su aplicación práctica son sometidos a una serie de cuestionamientos que tienen que ver con particularidades regionales y cronológicas.

$<$ Canastas de consumo $><$ Buenos Aires $><$ Precios $><$ Salarios $>$

\section{Abstract}

This paper analyses the methodology for the construction of a consumption basket for Buenos Aires households during the early decades after independence, based on models suitable for international comparisons. These models and their application to historical studies will be discussed here in relation to regional and chronological issues.

$$
<\text { Consumption baskets }><\text { Buenos Aires }><\text { Prices }><\text { Wages }>
$$

En el presente trabajo nos proponemos analizar y discutir las posibilidades y limitaciones que ofrece una metodología hoy muy empleada en los estudios sobre nivel de vida a escala internacional. Se trata de las propuestas formuladas inicialmente por Robert Allen (2001), quien creó un modelo para la construcción de canastas de consumo constituidas por un grupo limitado de bienes básicos para la vida y que se conforman con los más accesibles y baratos de cada sitio, de manera de poder establecer canastas comparables a nivel internacional que se puedan poner en relación con los ingresos salariales de ciertas categorías de trabajadores presentes en casi todos lados, como son los obreros de la construcción. Esta metodología ha permitido desarrollar estudios en muchos lugares del planeta y en diversos períodos, conformando una poderosa herramienta para la historia comparada de los niveles de vida. Sin embargo ella no deja de presentar una serie de problemas que son necesarios abordar al realizar este tipo de

\footnotetext{
* Doctor en Historia. Instituto Ravignani-UBA/CONICET. dvsantilli@gmail.com

** Doctor en Historia. Instituto Ravignani-UBA/CONICET. jorgegelman@gmail.com
} 
Santilli y Gelman. Los estudios sobre el nivel de vida. La metodología de la canasta aplicada a la primera mitad...

estudio. Ambas cuestiones: las posibilidades y las limitaciones de esta metodología son las que abordamos aquí, a través de su aplicación al caso porteño en la primera mitad del siglo XIX.

Una de las diversas formas de medir el nivel de vida de una sociedad dada es el estudio de los bienes de todo tipo que consume. Desde los más elementales, los alimentos, hasta el modo en que se divierte o descansa forman parte del consumo medible para establecer una pauta sobre el nivel de vida. En nuestras sociedades contemporáneas estos estudios son encarados por el Estado en forma permanente, por dependencias conformadas específicamente a tal efecto. Desde ya que las informaciones que se ofrecen generan controversias interesadas desde todos los ámbitos, privados y públicos, pero no hay que olvidar que las mismas no dejan de ser estadísticas, por lo tanto, son una generalización que contempla como mucho la moda, es decir lo más común. ${ }^{1}$

Desde el punto de vista de nuestra materia, la historia, las estadísticas referidas al consumo comienzan, como muy atrás, bien avanzada la primera mitad del siglo XX en los países centrales. En nuestro país la primera estimación oficial del costo de la canasta de consumo de un matrimonio con dos hijos menores se publicó en 1933 (Departamento Nacional del Trabajo, 1933). ${ }^{2}$ Sin embargo, el concepto de canasta básica recién se incorpora a la estadística oficial en 1988, incluyendo el método de la línea de pobreza ${ }^{3}$. Es evidente que la información que nos puedan brindar las estadísticas oficiales no nos sirven a menos que nuestro arco temporal de estudio se limite a las últimas décadas del siglo XX.

Pero lo que nos proporciona el conocimiento de la metodología empleada actualmente es su posible aplicación a la historia previa. Claro que para ello hay que reconstruir la materia básica con la que se elabora en la actualidad la canasta de consumo de los pobladores. Es decir, el conjunto de bienes y servicios necesarios para la mayoría de la población y un índice de precios minoristas que permita asignarle un valor a aquéllos y otro de salarios, así como de otros posibles ingresos de la población considerada. ¿Es posible reconstruir tales datos en períodos históricos concretos? Ello dependerá de diversas variables, no siendo la menos importante, aunque la más obvia, la lejanía del período que vamos a estudiar. Cuanto más atrás nos vamos en el tiempo suele ser más insegura una buena parte de los datos obtenidos. En nuestro caso nos centraremos en la primera mitad del siglo XIX.

La reconstrucción de la canasta de consumo de las familias requiere de una investigación que supera en principio el marco cuantitativo. Si esa información puede obtenerse a través de archivos en los que se describe la compra de artículos de consumo, sean de entidades públicas (ejército, hospitales), semipúblicas (conventos, colegios) o privadas (testamentarias), ellas deben pasar por un severo cedazo que analice el proceso

1 Sobre la metodología y práctica de la elaboración de canastas de consumo en la actualidad en la Argentina puede verse (Gasparini, Cicowiez, \& Sosa Escudero, 2013) (Pizzolitto, 2007).

2 Citado en (Instituto Nacional de Estadística y Censos, 2012).

3 Para una discusión sobre la metodología de la medición de la pobreza, véase (Olavarría Gambi, 2001) y (Boltvinik, 1990), entre otros. Para el caso argentino, véase (Velazquez (dir.), 2016). 
de construcción de la fuente para asegurar su credibilidad. Por ejemplo, para señalar una obviedad, si se trata de información de entidades públicas, debe tratar de verificarse que los precios y las cantidades que está adquiriendo el Estado son compatibles con la cantidad de usuarios de esos bienes o servicios, y si los precios se corresponden con los de mercado. En el caso de conventos o colegios que dependían de órdenes religiosas, muchas veces se producían algunos alimentos en sus propias instalaciones o en dependencias productivas de las mismas, por lo que lo que compraban no era la totalidad del consumo; también hay que tener cuidado con las compras especulativas, ante un probable aumento de precios por diversas razones o, si lo que analizamos son los pagos realizados, es necesario verificar que no se estén acumulando saldos deudores de períodos mayores. Asimismo, es conveniente contar con el universo de las bocas a alimentar, de manera de ponderar el consumo observado. Por último, las contabilidades particulares suelen ser más ricas, pero muy difíciles de conseguir. En el caso de las testamentarias, las fuentes privadas más accesibles para esos períodos remotos, no suelen incluir los bienes básicos de consumo alimenticio, que son de lejos la parte principal de las necesidades de una familia de los sectores populares. Pueden ser fuentes de gran utilidad para estudiar los consumos comparados de diversos grupos sociales y su evolución en el tiempo, pero muy poco nos pueden informar del consumo de las necesidades básicas de la población.

Pero además, y creemos que este es el punto esencial de la elaboración de la canasta, debe contextualizarse con un espíritu crítico riguroso, labor que es esencial para los historiadores, conocedores del lapso y la región que se está analizando. Los productos que se consumen, así como sus cantidades y precios, deben ser evaluados a la luz de ese conocimiento del contexto histórico. Es decir, se debe tener en cuenta si el producto que los libros indican que era consumido es mencionado en otras fuentes, cuantitativas o no, que complementan la información anterior, como las cartas privadas entre contemporáneos o las descripciones de los testimonios de viajeros. Por último, debe tenerse el convencimiento de que lo consumido en esa institución era posible en tal espacio y refleja medianamente el modo de vida de los habitantes de la región.

\section{La metodología en discusión}

Para la época que estudiamos contamos básicamente con los registros de instituciones que dependían de órdenes religiosas, sean conventos, hospitales o colegios, así como del estado, por ejemplo, los gastos para el sostenimiento de cuerpos militares. En general, las primeras, las más utilizadas, registraban las salidas de dinero por todo concepto, que son las que nos interesan, siempre que mencionen la cantidad adquirida para cada ítem consumido. Si este dato no está, situación bastante común, un procedimiento posible es reconstruir las cantidades con series de precios obtenidos en otras fuentes. Dependiendo de la calidad de la información con la que se cuente se podrá procesar la totalidad de la fuente o una muestra que no puede ser inferior a un año. Este lapso anual debe ser la medida mínima y el corte de toda la información, para poder 
Santilli y Gelman. Los estudios sobre el nivel de vida. La metodología de la canasta aplicada a la primera mitad...

establecer estacionalidad en las compras, además de poder comparar entre varios años para percibir situaciones no comunes, como adquisiciones especiales.

Una consideración que se agrega a la anterior es si la fuente informa sobre la cantidad de personas a las cuales están destinadas esas compras. Si contamos con ese dato, podremos construir lo que denominamos una canasta individual real, dividiendo las cantidades totales compradas por la cantidad de personas que las consumen. Esta es la situación ideal; como ejemplo podemos mencionar el caso que estudiaron Moraes y Thul (2015), quienes accedieron a una lista del presupuesto de compras de una compañía de portuarios de fines de la colonia, con la nómina de integrantes de la misma.

Pero también puede suceder que no se cuente con la cantidad de consumidores por las compras. En ese caso deberán establecerse los porcentajes de incidencia de cada producto en la compra total. Con ello se construye una canasta mínima de consumo individual, partiendo de la base de un piso de alimentos necesarios para la reproducción humana. Un ejemplo de esta canasta es la conocida como Bare Bone Basket (BBB), propuesta por Robert Allen (2001) hace unos años, e ideada con la finalidad de poder generalizarse para diversas geografías y momentos de la historia, de manera de poder comparar los niveles salariales 'reales' a partir de los ingresos necesarios en cada lugar y momento para alcanzar ese consumo mínimo de calorías y bienes.

La canasta real mencionada, que se obtiene a partir de fuentes históricas, puede compararse con esta mínima para establecer cuál es su distancia con respecto al mínimo de subsistencia. Es un ejercicio que dará como resultado una apreciación del nivel de vida de la sociedad en estudio de manera comparada y que por lo tanto permitirá realizar un conjunto de análisis referidos por ejemplo a los niveles de desarrollo económico de cada lugar en relación al resto, o sobre las presiones migratorias, etc.

Hemos utilizado ya la técnica de $B B B$ para reconstruir la canasta de Buenos Aires en la primera mitad del siglo XIX (Gelman \& Santilli, 2016). Contábamos con las compras del Hospital de mujeres para una buena parte de los años que van de 1820 a 1850 . De esa treintena elegimos tres por la relativa tranquilidad económicas del momento; 1825 , justo antes del cimbronazo inflacionario provocado por el bloqueo a Buenos Aires de la flota brasileña y la emisión de moneda inconvertible; 1835, año en que las principales variables económicas están más o menos serenas en Buenos Aires, ya que no hay guerras o mayores conflictos sociales en su territorio, ni tampoco inclemencias del tiempo o epidemias; y por último 1849, cuando la economía está creciendo por el incremento de las exportaciones en los últimos años del lapso que la historiografía ha llamado la pax rosista. Comprobamos además que no hubiera compras excesivas de algún artículo; por ejemplo, hallamos que en diciembre de 1825 se adquirieron cantidades de vino muy por encima del posible consumo o de la compra de ese bien en otros años, concluyendo que era probable que se tratara de adquisiciones de tipo especulativo ante el cierre de las importaciones por el bloqueo brasileño y la inminente escalada inflacionaria. También tuvimos que desechar algunas compras porque no contaban con las cantidades del producto adquirido, pero concluimos que su incidencia en el consumo era relativamente menor. Por ejemplo, las verduras compradas en el 
mercado. Además, dado el tipo de fuente que utilizamos, un hospital, algunos artículos consumidos a nivel popular no estaban presentes, al menos en proporciones similares a los de esta población. Ese podría ser el caso de las bebidas alcohólicas, cuyo consumo incorporamos a través de las introducciones desde Cuyo a este mercado y considerando la población de Buenos Aires pasible de la ingesta de alcohol. A la inversa, debimos deducir del cálculo aquéllos bienes específicos de una institución asistencial, como los medicamentos.

Obtuvimos así, luego de una serie de pasos que permitieron llevar el consumo del hospital al de una persona cuyo consumo equivale al de las calorías establecidas por la bibliografía de referencia como el mínimo indispensable para su supervivencia, una canasta que representaba el nivel de subsistencia de una familia tipo compuesta por un matrimonio con dos hijos, susceptible de ser comparada con otras canastas construidas para Buenos Aires y para otras regiones del mundo, tal como lo plantean los autores que proponen este tipo de metodología. Se podría decir, parangonando con los estudios estadísticos sobre la actualidad, que marcamos una especie de línea de indigencia, por debajo de la cual era muy difícil sobrevivir en ese entonces. En el cuadro siguiente se puede ver cuál es la composición de dicha canasta para un adulto en 1835 .

Tabla 1. Composición de la canasta BBB - Buenos Aires, 1835

\begin{tabular}{ccccc}
\hline & Calorías diarias & Proteínas & $\mathrm{Kg} / \mathrm{l}$ por mes & Valor en $\$$ fuertes \\
\cline { 2 - 5 } Arroz & 32 & 0 & 0,281 & 0,21 \\
Pan & 443 & 12 & 5,426 & 3,54 \\
Azúcar & 36 & 0 & 0,280 & 0,31 \\
Carne & 1241 & 64 & 14,890 & 2,93 \\
Cerveza & 0 & 0 & 0,003 & 0,00 \\
Chocolate & 19 & 0 & 0,099 & 0,25 \\
Fideos & 25 & 1 & 0,207 & 0,17 \\
Gallina & 14 & 1 & 0,242 & 0,14 \\
Grasa & 38 & 0 & 0,131 & 0,17 \\
Leche & 47 & 0 & 2,465 & 0,63 \\
Pollo & 13 & 1 & 0,231 & 0,08 \\
Poroto & 16 & 1 & 0,144 & 0,03 \\
Vino & 11 & & 0,399 & 0,20 \\
Aguardiente & 5 & & 0,192 & 0,27 \\
Total & 1941 & 80 & & 8,95 \\
\hline
\end{tabular}

Fuente: (Gelman \& Santilli, 2016)

Se puede apreciar la relativa amplitud del consumo de los pobladores de Buenos Aires por esos años, desde carne, básica en la región, hasta chocolate, pasando por azúcar, arroz y vino. Esta riqueza es rescatable cuando la comparamos con lo mostrado 
Santilli y Gelman. Los estudios sobre el nivel de vida. La metodología de la canasta aplicada a la primera mitad...

para otros espacios. De todos modos, quedó afuera de este cálculo la incidencia del alquiler si hablamos de la ciudad, pero como hemos comparado con elaboraciones para otras ciudades que tampoco la tuvieron en cuenta nuestro cálculo no pierde validez. Evidentemente se plantean algunos problemas en la canasta obtenida del hospital, sobre todo por el hecho de que es posible que ciertos consumos, como podría ser el caso del chocolate, fueran específicos de una institución de este estilo y no estuvieran incluidos en la dieta ordinaria de los pobladores pobres de la región. Sea como sea, se puede ver que la incidencia que este tipo de bien tiene en nuestro cálculo es poco relevante. Y lo que salta a la vista es el peso extraordinario que tenían dos bienes, la carne y el pan, en el valor de la canasta y en las calorías ingeridas.

Si bien este sistema es muy eficaz para la comparación entre distintas regiones y momentos, una serie de críticas se le han hecho, que compartimos en general ${ }^{4}$. En primer lugar, la cantidad de calorías necesarias para la mínima supervivencia del ser humano. Si bien no parece descabellada la cifra de 2000 calorías diarias para un adulto, siempre que se tome como un mínimo indispensable, es indudable que juegan otros factores en la determinación de ese mínimo, desde la ubicación geográfica, difiriendo de regiones frías o cálidas, del tipo de trabajo que se realiza, dependiendo del desgaste, de la edad y del sexo. ${ }^{5}$ También es necesario tener en cuenta la etapa histórica, si bien podemos suponer que la humanidad no ha cambiado tanto en los años que estamos estudiando. ${ }^{6}$

Otra cuestión que se debate es la de la cantidad de componentes de la casa de familia, que es la unidad básica de medición. Es decir, lo que se mide es el consumo de la familia, cuyos componentes pueden variar de lugar en lugar y por etapas históricas, y considerando únicamente el aporte del jefe o jefa en los ingresos. En primer lugar, es discutible que en economías donde la unidad familiar es no solo la unidad reproductiva y de convivencia sino además la unidad económica básica de producción, sólo aporte el jefe de familia. Sabemos del trabajo infantil en situaciones donde aún las regulaciones legales no han llegado a limitarlo. Y sabemos del trabajo de la mujer, ya sea en contextos rurales, pero también urbanos, en tareas que se consideraban típicamente femeninas; lavanderas, planchadoras, costureras, etc. Por último, pero no menos importante, es la relacionada con la cantidad de componentes de la unidad familiar. Se considera que la familia estaba compuesta, a los efectos del cálculo del consumo mínimo indispensable, por el jefe, que equivale a una unidad, la mujer, cuyo coeficiente de consumo frente a la unidad es de 0.80, y la de dos menores de diferentes edades que consumen, sumados, 1.2 unidades. Todo esto suma tres unidades, es decir una canasta mínima de 1941 calorías diarias multiplicadas por 3, o sea 5.823 calorías diarias.

\footnotetext{
${ }^{4}$ Sobre las críticas generales véase por ejemplo (Dobado-Gonzalez, 2015a) y las respuestas que originó en el mismo número 33 de la Revista de Historia Económica.

5 Véase Dietary Reference Intakes for Energy, Carbohydrate, Fibber, Fat, Fatty Acids, Cholesterol, Protein, and Amino Acids (2002), en http://www.uned.es/pea-nutricion-y-dietetica-I/guia/index.htm, citado por (Gonzalez Mariscal, 2015)

${ }_{6}$ Robert Allen (2001) basa su apreciación de 1941 calorías en (Fogel, 1991).
} 
Más allá de la valoración de los coeficientes de cada miembro de esa familia ${ }^{7}$, una cuestión discutible es la cantidad de componentes, impertérrita a través de los siglos y de las geografías. Tanto en China como en Japón o la India del siglo XVIII la cantidad de integrantes de la familia está considerada del mismo modo que en Londres o Leipzig para la misma fecha, 3 (Allen, Bassino, Ma, Moll-Murata, \& Van Zanden, 2011). El mismo criterio se aplica a las sociedades americanas sin siquiera discutirlo (Arroyo Abad, 2013b). Basta revisar el desarrollo de la demografía histórica para verificar que estas cifras son irreales, que no tenían la misma cantidad de componentes familiares una casa de Pekín que una de Londres; ni siquiera una ubicada del otro lado de la línea diseñada por John Hajnal (1965) en Europa, por ejemplo, Varsovia, que una de Amberes. En el caso de América, esa cantidad de componentes peca de escasa también. En definitiva, creemos que la magnitud de 3 habitantes por unidad familiar sólo puede ser usada a título comparativo y haciendo la salvedad de tal situación. ${ }^{8}$

Otra cuestión que se agrega a la discusión es la composición de la canasta. Si bien Allen reconoce la variación de dicha composición de acuerdo con la producción de cada espacio, algunos de sus seguidores que aplicaron la fórmula inicial no lo tienen en cuenta. Es así como se calculan alimentos cuya producción no era la más importante ni su consumo el más popular, como el caso del trigo en Buenos Aires en detrimento de la carne (Arroyo Abad, 2013b), o se desconoce el aporte calórico de ciertos alimentos y bebidas a la dieta, como muestra Andrés Calderón (2015) para la ciudad de México.

También importa la calidad de los alimentos. Un estudio reciente comprueba que durante buena parte de la edad moderna se utilizó para la elaboración de pan una calidad de cereal más económico ("el efecto avena", lo denominan los autores) no tenido en cuenta por los formuladores de la metodología (López Losa \& Piquero Zarauz, 2016). Asimismo, en una primera instancia no se incorporaba el costo del alquiler de la vivienda en los ámbitos ciudadanos, aunque luego fue corregido (Allen, Murphy, \& Schneider, 2015). En definitiva, se prioriza la posibilidad de comparación entre diversos espacios y a través del tiempo, para lo cual hace falta generalizar, en lugar de abogar por la verosimilitud de las propuestas. Y sin embargo es evidente que en ciertos contextos el gasto de una familia en alquiler, por ejemplo, puede ser un elemento marginal, mientras que en otros puede tener un peso importante para calcular el mínimo indispensable para la supervivencia.

Por último, la construcción de un coeficiente de bienestar (welfare ratio, WR en adelante), cuya fórmula es el producto del monto del salario dividido por la canasta básica familiar ${ }^{10}$, es decir la cantidad de canastas básicas que se pueden adquirir con el salario medido de diversas maneras, puede pecar de arbitraria. En primer lugar porque,

7 Discutida entre otros por González Mariscal (2015).

8 Sobre el aporte del trabajo de la mujer y de los hijos, así como de la cantidad de componentes y de calorías consumidas, véase el documentado trabajo de Jane Humphries (2011), para la Inglaterra de fines del siglo XVIII.

9 Para considerar el alquiler, se estimó en un 5\% más el costo de la canasta básica.

${ }^{10}$ Para establecer la canasta básica familiar, además del costo de las 1941 calorías, se le adiciona el 5\% más per capita para considerar el alquiler, el costo del jabón, lumbre y combustible, más un adicional por ropa. 
Santilli y Gelman. Los estudios sobre el nivel de vida. La metodología de la canasta aplicada a la primera mitad...

con la finalidad de ser comparativa, se utiliza el salario del peón de albañil. Debería tenerse en cuenta qué grado de representatividad tiene tal salario en el conjunto de los percibidos por trabajadores de la ciudad motivo de la investigación, tal como aconseja Allen $(2001,414)$. En principio debería tenerse en cuenta si los trabajos de albañilería están dentro de la demanda global de trabajadores de la ciudad, es decir si no están sobre demandados o sobre ofertados con respecto a otras actividades ${ }^{11}$. Es muy probable que, si los alquileres son altos, también los salarios lo sean, ya que lo primero implica una demanda de habitación por encima de la oferta, situación que se traslada seguramente a la construcción. Esto se subsanaría con una evaluación de la composición de la masa laboral para establecer un promedio ponderado de su salario. Asimismo, como las remuneraciones son en general diarias, se fija arbitrariamente en 250 la cantidad de días trabajados por año, que Allen justifica comparando diversos casos presentados en la bibliografía europea $(2001,425)$. Puede pensarse que en diferentes ciudades la cantidad de días trabajados puede variar según las leyes de mercado, las costumbres, las remuneraciones, etc. y ello no invalida la posibilidad de comparar, ya que el salario será menos o más alto y hablará del nivel de ingresos reales.

Asimismo, como estamos mostrando, sólo se tiene en cuenta el consumo y el salario urbano, en sociedades donde la mayor parte de la población todavía vive de actividades rurales como bien marcan para el caso mexicano el trabajo de Jáuregui y Marichal (2015). La consideración de la canasta rural deberá tener en cuenta, además, la provisión de bienes no adquiridos en el mercado, es decir entregados como parte de las remuneraciones o aportados por actividades no reguladas por el mercado. De ese modo se valoriza con mayor verosimilitud la incidencia del salario en el total de ingresos de la familia rural. ${ }^{12}$

Una última reflexión que atañe al resultado final. Debe comprobarse la verosimilitud del coeficiente de bienestar logrado. Una $W R$ demasiado alta debe obligarnos a revisar, no sólo la construcción física de la misma, sino la posibilidad fáctica de su aplicación. Para que ello ocurra debe haber un crecimiento de la economía que pueda comprobarse por otros medios y una distribución funcional del ingreso y la riqueza que lo haga posible. A la inversa, si la tasa es negativa y se mantiene durante mucho tiempo, debemos revisar la posibilidad fáctica de que esto suceda. Es imposible que una sociedad se reproduzca si no puede consumir lo mínimo indispensable para ello durante mucho tiempo. Tal situación implica pensar, en primer lugar, en un error de la construcción, y en segundo lugar, una vez descartado el error, en cómo hicieron para sobrevivir. Se debe suponer que completaron su dieta con algún otro ingreso que no puede ser medido desde este herramental. ${ }^{13}$

${ }^{11}$ Indudablemente, la construcción está íntimamente relacionada con el crecimiento de la ciudad en cuestión. Así por ejemplo en el caso de la ciudad de Cochabamba, en Bolivia, cuyo crecimiento demográfico a través de un siglo fue muy escaso, puede deducirse una también escasa actividad de construcción y por consecuencia salarios bajos en ese rubro (Henriques, 2016).

${ }^{12}$ Una muy interesante reflexión sobre tal participación en (Djenderedjian \& Martirén, 2015).

${ }^{13}$ Un ejemplo de esta elaboración no revisada en (Arroyo Abad, 2013a). 
De todos modos, consideramos que la metodología desarrollada por estos investigadores es por ahora la mejor opción para este tipo de análisis en sociedades del pasado, sobre las que tenemos datos fragmentarios y muy pocas posibilidades de lograr series continúas. Asimismo, es la que nos permite comparar con otras sociedades lejanas en tiempo y espacio, y profundizar así la posibilidad de diálogo con otras investigaciones.

\section{EI análisis puntual comparativo}

Nuestro estudio puntual del caso de Buenos Aires, parte del cual hemos mostrado en el cuadro 1, nos llevó a calcular una $W R$ para el peón de albañil de la ciudad, trabajando 250 días al año, de 2.23 para $1825,1.50$ para 1835 y de 3.51 para 1849 (Gelman \& Santilli, 2016). Este cálculo difiere notablemente del elaborado por Leticia Arroyo Abad para la etapa de fines de la colonia y primera época independiente. La autora concluye que en Buenos Aires el coeficiente de bienestar era, entre 1775 y 1820, de 9.47 (2011, pág. p. 9. Table 5), y de 5.22 en 1830 (2013a, pág. 132). Se trata de un resultado que colocaba a Buenos Aires entre los sitios con el salario más elevado a nivel internacional en capacidad de compra de la canasta básica. Puede notarse que la diferencia con nuestros resultados es lo suficientemente alta como para que amerite control y comprobación detallados, de acuerdo con lo formulado en páginas previas acerca de la verosimilitud de las conclusiones obtenidas.

En principio se puede señalar que la canasta construida por Arroyo Abad et al. difiere en buena parte con la nuestra. El trigo proporcionaba 1218 calorías diarias y la carne 719 , según su cálculo Es decir $63 \%$ para el trigo (pan) y $37 \%$ para la carne ${ }^{14}$, mientras que nuestro cálculo, según se aprecia en el cuadro 1, indica que de las 1941 calorías 1341, el 64\%, corresponden a carne y 443 , el $23 \%$, al pan. Desde ya que las proporciones de estos dos bienes alimenticios básicos utilizada por estos autores no nos parecen acordes a los diversos estudios que se refieren a la región y que, todos, han señalado el peso de la carne en el consumo local, dada su abundancia y baratura relativa, desde relatos impresionistas contemporáneos, como el de Félix de Azara (1850), a estudios cuantitativos más recientes como los de Garavaglia (1994). Al observar la base de datos utilizada por estos autores en el trabajo de $2011^{15}$, pudimos comparar más en detalle sus cálculos. El primer acercamiento es verificar que tienen datos de $W R$ para los lapsos 1775/1811 y luego 1827/1860. Para el primero de los períodos, según la base utilizada, la $W R$ sería de 6.82. Y para 1830 es de 3.44 (solapa Argentina wages en la base). Esa canasta, como dijimos, se compone para todo el período en análisis sólo de carne y trigo, a lo que se le agrega un costo adicional de jabón, lumbre y combustible estimado en un $34 \%$ cuando no consta el precio, porcentaje que se reduce cuando la

\footnotetext{
${ }^{14}$ http://gpih.ucdavis.edu/Datafilelist.htm\#Latam. Consultada el 3/7/2014. El archivo en Excel se denomina "prices-wages-argentina-bolivia". Agradecemos a Tomás Guzmán el habernos advertido de su existencia.

15 Ídem nota anterior.
} 
Santilli y Gelman. Los estudios sobre el nivel de vida. La metodología de la canasta aplicada a la primera mitad...

base contiene el precio de alguno de esos $\operatorname{productos}^{16}$. Nuestra canasta en ese sentido es más amplia ya que incorpora otros bienes alimenticios, como arroz y azúcar, porotos, chocolate, fideos y leche, además de vino y aguardiente, excluyendo únicamente el costo de la vivienda.

Pero la mayor diferencia se verifica en los salarios; nuestra $W R$ está construida teniendo en cuenta el salario del peón albañil tal como lo estipula la metodología de referencia ${ }^{17}$-más allá de las reservas que marcamos-, en el caso del trabajo que estamos analizando, se toman otros valores. Para el lapso 1775-1809 los autores utilizan el salario del albañil, obtenido del trabajo de Lyman Johnson (Johnson \& Tandeter, $1990)^{18}$. Estos datos figuran en otro trabajo del mismo autor donde la remuneración del albañil era de 8 reales diarios en 1770, 4 reales a partir de 1773, subiendo a 5 en 1807 (Johnson, 1990, págs. cuadro 1, pp 138/9). Si se considera que los autores calculan 200 los días trabajados ${ }^{19}$ al año, para llegar a los 204 pesos que estiman, usaron para todo el período el valor más alto es decir 8 reales ( $8 \mathrm{r}$ x 200/8r por peso), que sólo lo percibieron según la fuente durante los primeros cinco años del lapso, bajando luego a la mitad. Hacia fines del mismo periodo, 1809, la cifra manejada por los autores es de $\$ 382.80$ al año, que equivale a 15 reales diarios, según los 200 días considerados como trabajados anualmente. En valores mensuales, equivale a \$37.50, mientras que un peón rural, según Johnson (1990), arañaba los \$10 y un marinero los \$13.- por mes. El salario del albañil, a razón de 5 reales diarios según Johnson, equivaldría a 10 ó 13 pesos mensuales, según se calcule a 200 o 250 días trabajados al año...

A partir de 1810, el salario que consideran es el de portero del gobierno central, tomado del trabajo de Barba (1999). No vamos a discutir la decisión de los autores acerca del tipo de sueldo que consideraron y de la representatividad del mismo en Buenos Aires. Pero evidentemente difiere notablemente del nuestro que es el peón de albañil. Nuestro trabajador urbano ganaba 5 reales al día, el portero de Arroyo Abad et al. ganaba \$33 al mes en 1810 (\$ 125.- y 396.- anuales respectivamente); en 1827, año informado por los autores más cercano a nuestro $1825^{20}$ el albañil percibía $\$ 1.40$ por día y el otro $\$ 25$ por mes ( $\$ 280$ y 300.- anuales respectivamente ${ }^{21}$ ). En 1835 , nuestro año base, el salario del peón de albañil era de $\$ 2.50$ por día y el del peón rural mensual era de $\$ 37.95$, promediando 73 casos encontrados, y el del portero era de $\$ 66$.- (anualizado, equivalía a $\$ 500,455.40$ y 799.20 , respectivamente). ${ }^{22}$

\footnotetext{
${ }^{16}$ Los autores no incluyen ningún adicional por ropa.

${ }^{17}$ Aceptamos ese criterio al sólo efecto de hacer nuestro cálculo comparable.

${ }^{18}$ No hemos tenido acceso a la versión original citada, pero en la versión en castellano (Johnson \& Tandeter, 1992) no constan los salarios citados. Es probable que la versión inglesa esté más completa.

${ }^{19}$ Se diferencian de la metodología sugerida por Allen al calcular el salario por 200 días trabajados. Si nosotros utilizáramos el mismo criterio, la diferencia con el resultado de Arroyo Abad et alii sería mayor: $1825,1.78 ; 1835,1.20 ; 1849,2.81$.

${ }^{20}$ Debemos mencionar que los autores no tienen datos, o no informan en la base, para el lapso 1812-1826.

${ }^{21}$ Recordemos que Arroyo Abad multiplica por 200 días al año y no por 250 como Allen.

${ }^{22}$ Ver la base "prices-wages-argentina-bolivia", solapa "Argentina wages" para todo este párrafo, mencionada en nota 14.
} 
De manera que, utilizando el salario del portero, el cálculo de los autores citados arroja un coeficiente de 3.16 para 1835. Si reemplazamos en la base de Arroyo et al. dicho salario por el de nuestro peón de albañil (\$2.50 por día), la $W R$ pasa a ser de 1.98 trabajando 200 días y de 2.47 por 250 días trabajados al año, siempre más bajo que el obtenido por los autores mencionados. Incluso en 1810, cuando la tasa para los autores es de 4.4, con el sueldo corregido del albañil según Lyman Johnson (1990) pasaría a ser de 1.74 .

Deducimos entonces que, más allá de las diferencias ya marcadas sobre la composición de la canasta, de la proporción de los alimentos, de la variedad de los mismos, de la relación entre lumbre y combustible con las calorías, etc., la diferencia entre el salario tomado explica la mayor parte de las divergencias. De modo que se confirma entonces nuestra reserva con respecto a los cuidados y precauciones que deben observarse con los datos para construir canastas y $W R$.

En síntesis, lo que hemos intentado mostrar en este breve texto son las dificultades y posibilidades que tenemos para construir canastas de consumo razonables para las distintas sociedades históricas, de manera de elaborar series de salarios reales e ingresos, que sean consistentes para analizar esa sociedad, pero que a la vez permitan ponerla en comparación con otras etapas de la misma o con otras contemporáneas. Para comenzar, en el caso argentino, es necesario construir estas series para todo el territorio y en el largo plazo, lo que sería fundamental para comprender la evolución comparada de sus regiones, la desigualdad que sin duda parece crecer de manera exponencial luego del proceso independentista, y que promueve, entre otras cosas, la agudización de los movimientos migratorios entre regiones con niveles de vida muy diversos. Obviamente esta no es la única variable que se debe considerar para comprender estos fenómenos, pero sí es un elemento central a tener en cuenta.

Igualmente estos estudios ayudarán a poner nuestra agenda de investigaciones en los debates internacionales sobre los niveles de vida y de desarrollo, aunque como hemos intentado mostrar, para que ello se haga eficazmente, se deben acrecentar las precauciones metodológicas y también la búsqueda de la información 'dura' que nos permita limitar al máximo la cantidad de supuestos que este tipo de trabajo necesariamente incluye y que pueden llevar a veces a resultados por demás extravagantes, por no decir alejados de la realidad.

\section{Referencias Bibliográficas}

Allen, R. C. 2001. The Great Divergence in European Wages and Prices from the Middle Ages to the first World War. Explorations in Economic History (38), 411-447.

Allen, R. C., Bassino, J.-P., Ma, D., Moll-Murata, C., \& Van Zanden, J. L. 2011. Wages, prices, and living standards in China, 1738-1925: in comparison with Europe, Japan, and India. The Economic History Review, 64(S1), 8-38.

Allen, R. C., Murphy, T. E., \& Schneider, E. B. 2015. Una de cal y otra de arena: Building Comparable Real Wages in a Global Perspectiva. Revista de Historia Económica / Journal of Iberian and Latin American Economic History, 33(1), 61-76. 
Santilli y Gelman. Los estudios sobre el nivel de vida. La metodología de la canasta aplicada a la primera mitad...

Arroyo Abad, L. 2013a. Inestabilidad, costo de vida y salarios reales en Venezuela en el siglo XIX. América Latina en la Historia Económica, 20(3), 114-137.

Arroyo Abad, L. 2013b. Persistent Inequality? Trade, Factor Endowments, and Inequality in Republican Latin America. Journal of Economic History, 73(1), 38-78.

Arroyo Abad, L., Davies, E., \& van Zanden, J. L. 2011. Between conquest and independence: Real wages and demographic change in Spanish America, 15301820. CGEH Working Papers(20), 1-49. doi:10.1016/j.eeh.2011.12.001

Azara, F. d. (1850). Viajes por la America del Sur. Montevideo: Imprenta del Comercio del Plata.

Barba, F. 1999. Aproximación al estudio de los precios y salarios en Buenos Aires desde fines del siglo XVIII hasta 1860. La Plata: Ediciones UNLP.

Boltvinik, J. 1990. Pobreza y necesidades básicas. Concepto y métodos de medición. Caracas: PNUD.

Calderón Fernández, A. 2015. Las canastas 'barebone' comparadas con las cestas globales de la compra y las dietas carcelarias en la ciudad de México en el siglo XVIII. XVII Congreso Mundial de Historia Económica. Kyoto.

Departamento Nacional del Trabajo. 1933. Costo de la Vida. Elaboración de Presupuestos Familiares. Determinación de números índices. Buenos Aires: DNT.

Djenderedjian, J., \& Martirén, J. L. 2015. Are salaries a so useful tool to build up comparable standards of living? Some caveats concerning salary elements, available currencies, debts and credit in pre-modern Rio de la Plata region, 17701830. XVII World Economic History Congress (WEHC). Kyoto.

Dobado González, R. G. 2015b. El bienestar económico y biológico en la América borbónica: una comparación internacional de salarios y estaturas. In J. Gelman, E. Llopis, \& C. Marichal (coords.), Iberoamérica y España antes de las independencias, 1700-1820. Crecimiento, reformas y crisis. México: Instituto Mora, El Colegio de México, 481-524.

Dobado-Gonzalez, R. 2015a. Pre-Independence Spanish Americans: Poor, Short and Unequal... Or the Opposite? Revista de Historia Económica - Journal of Iberian and Latin American Economy History, 33(1), 15-60.

Fogel, R. W. 1991. The Conquest of High Mortality and Hunger in Europe and America: Timing and Mechanisms. In P. Higonnet, D. S. Landes, \& H. Rosovsky (eds.), Favorites of Fortune: Technology, Growth, and Economic Development since the Industrial Revolution. (pp. 33-71). Cambridge: Harvard University Press.

Garavaglia, J. C. 1994. De la carne al cuero. Los mercados para los productos pecuarios (Buenos Aires y su campaña, 1700-1825). Anuario IEHS(9), 61-96.

Gasparini, L., Cicowiez, M., \& Sosa Escudero, W. (2013). Pobreza y desigualdad en América Latina. Conceptos, herramientas y aplicaciones. Buenos Aires: Temas/ CEDLAS.

Gelman, J., \& Santilli, D. 2016. ¿El paraíso de los asalariados? La canasta de consumo y el nivel de vida de los pobladores de Buenos Aires, siglso XVIII y XIX. $V$ Encontro rural Rcport - XV Congreso de Historia Agraria de la SEHA. Lisboa. 
Gonzalez Mariscal, M. 2015. Precios y niveles de vida en Sevilla durante la inflación del vellón. Una primera aproximación. XVIIth World Economic History Congress, Kyoto, 1-26, 3-7/8/2015.

Hajnal, J. 1965. European marriage pattern in historical perspective. In D. Glass, \& (. D. Eversley, Population in History. London: Arnold.

Henriques, R. 2016. Evolución de los niveles de vida y la desigualdad en el municipio de Cochabamba, 1825-1925. Lisboa: Ponencia presentada en le XV Congreso de Historia Agraria de la SEHA.

Humphries, J. 2011. The lure of aggregates and the pitfalls of the patriarchal perspective: a critique of the high wage economy interpretation of the British Industrial Revolution. Discussion Papers in Economic and Social History, University of Oxford., Oxford.

Instituto Nacional de Estadística y Censos. 2012. Canasta bäsica alimentaria y canasta básica total. Historia, forma de cálculo e interpretación. Buenos Aires: INDEC. Retrieved 04 10, 2016, from http:/www.indec.mecon.ar/ftp/nuevaweb/ cuadros/74/informe_canastas_basicas.pdf

Jauregui, L., \& Marichal, C. 2015. La economía mexicana desde la época borbónica hasta las guerras de Independencia, 1760-1810. In J. Gelman, E. Llopis, \& C. Marichal (coords.), Iberoamérica y España antes de las independencias, 17001820. México: Instituto Mora, El Colegio de México, 111-162.

Johnson, L. 1990. Salarios, precios y costo de vida en el Buenos Aires colonial tardío. Boletín del Instituto de Historia Argentina y Americana Dr. Emilio Ravignani(2), 133-157.

Johnson, L., \& Tandeter, E. 1992. Economías coloniales. Precios y salarios en América Latina, siglo XVIII. Buenso Aires: FCE.

Johnson, L., \& Tandeter, E. 1990. Essays on the Price History of Eighteenth-Century Latin America. Albuquerque: University of New Mexio Press.

López Losa, E., \& Piquero Zarauz, S. 2016. Spanish real wages in the North-Western European mirror, 1500-1800. On the timmings and magnitude of the little divergence in Europe. AEHE.

Moraes, M. I., \& Thul, F. 2015. Precios, salarios y costo de vida en el Río de la Plata: Montevideo, 1760-1810. Kyoto, Japón, 3-7 de agosto de 2015: Ponencia presentada en el XVII Congreso Mundial de Historia Económica (WEHC).

Olavarría Gambi, M. 2001. Pobreza: Conceptos y medidas. Santiago: Universidad de Chile. Instituto de Ciencia Política.

Pizzolitto, G. 2007. Curvas de Engel de Alimentos, Preferencias Heterogéneas y Características Demográficas de los Hogares: Estimaciones para Argentina. La Plata: CEDLAS.

Velazquez, G. A. (Dir.) 2016. Geografia y Calidad de vida en Argentina. Tandil: Univesidad Nacional de Centro. 